03,05,09

\title{
Фотоиндуцированный магнитооптический эффект Керра в сульфиде европия EuS
}

\author{
(С) П.А. Усачёв, В.Н. Кац, В.В. Павлов \\ Физико-технический институт им. А.Ф. Иофрфе РАН, \\ Санкт-Петербург, Россия \\ E-mail: usachev@mail.ioffe.ru
}

Поступила в Редакцию 26 марта 2020 г.

В окончательной редакции 26 марта 2020 г.

Принята к публикации 2 апреля 2020 г.

\begin{abstract}
Проведено исследование влияния светового воздействия на магнитооптический эффект Керра в ферромагнитном полупроводнике сульфиде европия EuS. Экспериментально установлено, что при воздействии на образец светом с энергией фотона больше ширины запрещённой зоны возникает фотоиндуцированная намагниченность, связанная с образованием магнитных поляронов с большим магнитным моментом около $3000 \mu$ В. Поляроны возбуждаются в узком диапазоне температур $12-18 \mathrm{~K}$ и образуют суперпарамагнитный ансамбль со средним временем жизни полярона $13 \mu \mathrm{s}$. Описывается способ наблюдения фотоиндуцированной намагниченности с помощью метода оптической накачки и зондирования.
\end{abstract}

Ключевые слова: магнитный полупроводник, фотоиндуцированная намагниченность, спиновый полярон, магнитооптический эффект Керра.

DOI: 10.21883/FTT.2020.09.49769.09H

\section{1. Введение}

Интерес к исследованию оптических свойств полупроводников и их связи с магнитными свойствами обусловлен как большой фундаментальной значимостью результатов, так и их возможным практическим применением для развития новых технологий, телекоммуникационных и вычислительных систем. Особый интерес вызывают магнитные полупроводники, поскольку они могут служить материальной платформой для спиновых фотонно-квантовых технологий [1], технологий сверхбыстрой магнитной записи [2] и бионанотехноглогий [3]. Халькогениды европия $\mathrm{EuX}(\mathrm{X}=\mathrm{O}, \mathrm{S}, \mathrm{Se}, \mathrm{Te})$ относятся к этой группе материалов и обладают рядом уникальных физических свойств [4]. Магнитные полупроводники $\mathrm{EuX}$ являются перспективными объектами для фундаментальных физических исследований $[5,6]$, и либо уже нашли [7], либо являются потенциально перспективными материалами для различных технических применений [8]. Будучи прозрачными в видимой или ближней инфракрасной области электромагнитного спектра, халькогениды европия $\mathrm{EuX}$ образуют кристаллы с гранецентрированной кубической структурой (точечная группа $m 3 m$ ) и ковалентным полярным типом химической связи. ЕuX могут быть интегрированы с различными подложками, такими как $\mathrm{GaN}$ [9], кремний [10], графен [11] или сложные оксиды [12]. Важной особенностью этих соединений является то, что они представляют собой группу собственных магнитных полупроводников - $\mathrm{EuO}$ и $\mathrm{EuS}$ обладают ферромагнитным типом упорядочения, EuTe имеет антиферромагнитный порядок, a EuSe является метамагнетиком со сложной магнитной фазовой диаграммой, включающей антиферромагнитный, ферри- и ферромагнитный типы упорядочения [4].

$\mathrm{B}$ магнитных полупроводниках $\mathrm{EuX}$ зона проводимости образована незаполненной $5 d$-оболочкой ионов $\mathrm{Eu}^{2+}$, а валентная зона сформирована заполненной $5 p$-оболочкой, на потолке которой находятся наполовину заполненные локализованные $4 f$-подуровни, имеющие семь электронов с суммарным спином 7/2. $\mathrm{EuX}$ являются соединениями с классическим гейзенберговским обменным взаимодействием [13], многие их особенности объясняются электронно-спиновыми состояниями [14], при этом энергия электронов минимальна при полном ферро- или антиферромагнитном упорядочении. В частности, в области критической температуры в EuX наблюдается сильный красный сдвиг края фундаментального поглощения [4]. Другой важной особенностью $\mathrm{EuX}$ является то, что электроны в зоне проводимости могут установить в своем окружении ферромагнитный тип магнитного упорядочения за счет сильного $d-f$ обменного взаимодействия $[15,16]$. Электроны в зоне проводимости, могут локализоваться в определенной области кристалла и образовать магнитные поляроны наноразмерные квазичастицы, состоящие из $4 f$-электронов с высокой степенью спиновой поляризации. При малых концентрациях электронов в зоне проводимости образование таких электронно-спиновых квазичастиц является энергетически выгодным, но не может повлиять на магнитное состояние кристалла в целом $[15,17,18]$. Одним из способов возбуждения электронов в зону проводимости полупроводника является воздействие на него светом с энергией фотонов больше ширины запрещенной зоны. В этом случае образование магнитных поляронов приводит к появлению фотоиндуцированной 
намагниченности, которая может быть зарегистрирована с помощью различных магнитооптических эффектов.

В настоящей работе мы сообщаем об экспериментальном исследовании фотоиндуцированного магнитооптического эффекта Керра в сульфиде европия EuS. Полученные экспериментальные данные однозначно доказывают возникновение фотовозбужденных магнитных поляронов с большим магнитным моментом. Установлено время жизни и диапазон температур, в котором существуют магнитные поляроны. Насколько нам известно, фотоиндуцированные магнитные поляроны в ферромагнитных полупроводниках до сих пор не наблюдались. Поэтому, обнаружение фотовозбужденных магнитных поляронов и определение условий, при которых они существуют, является важной и актуальной задачей.

\section{2. Методика эксперимента}

Исследование фотоиндуцированного магнитооптического эффекта Керра в объемном образце $\mathrm{EuS}$ проводилось с помощью метода оптической накачки и зондирования. Схема эксперимента представлена на рис. 1. Образец был помещен в оптический криостат замкнутого цикла, позволявший проводить измерения в диапазоне температур 3-300 K в геометрии полярного эффекта Керра. В данной геометрии внешнее магнитное поле приложено параллельно волновому вектору зондирующего луча, направленному по нормали к поверхности образца. Источником накачки служил $\mathrm{Yb}: \mathrm{KYW}$ лазер с длиной волны $1032 \mathrm{~nm}$ и нелинейным преобразователем для создания луча накачки с энергией фотонов $2.4 \mathrm{eV}$. Эта энергия превышает ширину запрещенной зоны EuS, составляющую $\sim 1.6 \mathrm{eV}$. Интенсивность излучения накачки модулировалась с частотой $1.46 \mathrm{kHz}$ либо механическим обтюратором, либо фотоупругим модулятором с возможными частотами модуляции $42 \mathrm{kHz}$ и $84 \mathrm{kHz}$. Луч накачки фокусировался на образец в пятно с гауссовым профилем и диаметром $\sim 300 \mu \mathrm{m}$. Линейно поляризованный зондирующий луч $\mathrm{He}-\mathrm{Ne}$ лазера с энергией фотонов $1.96 \mathrm{eV}$ и мощностью $\sim 1 \mathrm{~mW}$ фокусировался на образец в пятно с гауссовым профилем и диаметром $\sim 250 \mu \mathrm{m}$. Перекрытие лучей на образце контролировалось с помощью системы визуализации.

С помощью магнитооптического поляриметра измерялась эллиптичность отраженного от образца зондирующего луча, связанная с полярным эффектом Керра. Оптическая часть поляриметра состояла из четвертьволновой пластинки, призмы Волластона и балансного фотодетектора, сигнал с которого регистрировался синхронным усилителем, настроенным на частоту модуляции накачки. Для калибровки установки модулятор помещался в зондирующий луч и измерялся заданный сигнал керровской эллиптичности.

Фотоиндуцированный сигнал в зондирующем луче измерялся при модуляции интенсивности луча накачки. При закрытой накачке эффект Керра в зондирующем луче был обусловлен только намагниченностью ионов

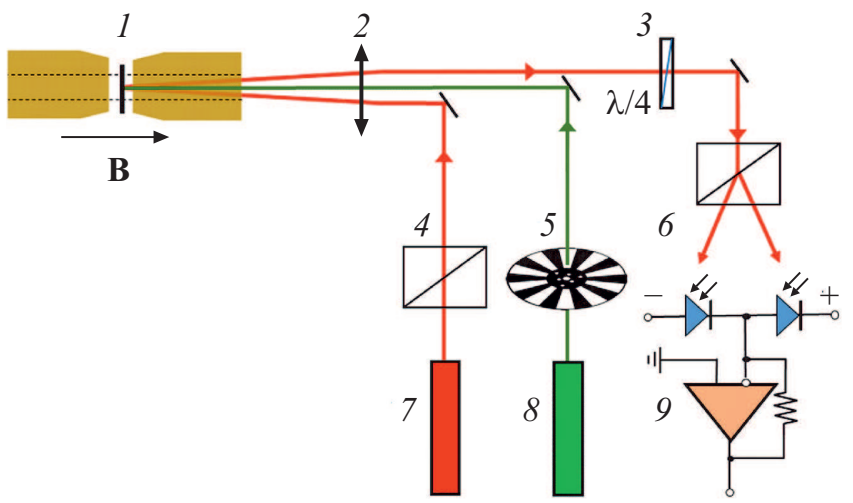

Рис. 1. Схема экспериментальной установки по наблюдению фотоиндуцированной намагниченности с помощью двухцветного метода оптической накачки и зондирования. Цифрами обозначены: 1 - образец в магнитном поле; 2 - фокусирующая линза; 3 - четвертьволновая пластинка; 4 - поляризатор; 5 - модулятор; 6 - призма Волластона; $7-$ зондирующий лазер; 8 - лазер накачки; $9-$ балансный фотодетектор.

$\mathrm{Eu}^{2+}$, а при воздействии накачки - намагниченностью невозбужденных ионов $\mathrm{Eu}^{2+}$ плюс фотоиндуцированной намагниченностью. После обработки сигнала методом синхронного детектирования оставался вклад только от фотоиндуцированной намагниченности. Доказательством того, что регистрируемый сигнал связан именно с воздействием накачки, служило то, что при отсутствии перекрытия лучей на образце фотоиндуцированный сигнал не наблюдался. Следует отметить, что наблюдаемый фотоиндуцированный сигнал содержал дополнительный вклад от теплового воздействия накачки на намагниченность невозбужденных ионов $\mathrm{Eu}^{2+}$, который рассматривался как паразитный и вычитался из наблюдаемого сигнала по процедуре, описанной в [19]. Во всех представленных далее экспериментальных результатах фигурирует фотоиндуцированный эффект Керра без теплового вклада, связанного с модуляцией намагниченности невозбужденных ионов $\mathrm{Eu}^{2+}$. В магнитном поле выше поля насыщения эффект Керра может составлять единицы градусов. Данная методика эксперимента позволяла с чувствительностью $\sim 1 \mu \mathrm{deg}$ измерять чистый фотоиндуцированный эффект.

\section{3. Результаты и обсуждение}

На рис. 2, а представлена зависимость фотоиндуцированной эллиптичности в эффекте Керра от внешнего магнитного поля В при мощности накачки $3.5 \mathrm{~mW}$. Данная зависимость имеет нелинейную $S$-образную форму, характерную для кривой намагничивания суперпарамагнитного ансамбля частиц. В нулевом магнитном поле фотоиндуцированный сигнал отсутствует, с увеличением поля фотоиндуцированный сигнал быстро насыщается. Это связано с тем, что фотоиндуцированные поляроны обладают большим магнитным моментом и их 

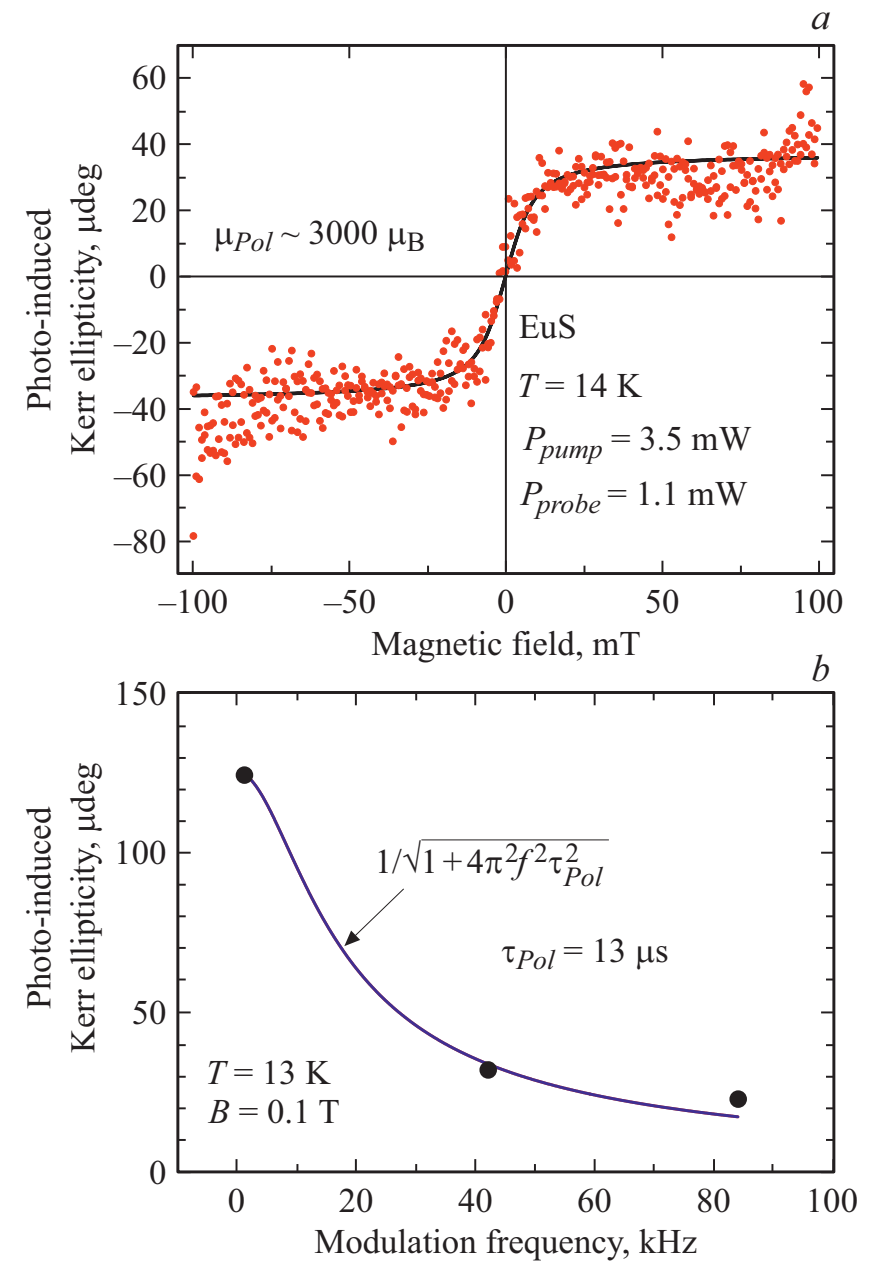

Рис. 2. Зависимость фотоиндуцированного эффекта Керра от магнитного поля $(a)$ и частоты модуляции $(b)$. Сплошные круглые точки - экспериментальные данные, линии - аппроксимация по уравнению (1) (a) и по функции, показанной на графике $(b)$, где $f-$ частота модуляции, $\tau_{P o l}-$ время жизни полярона.

ансамбль насыщается уже в небольшом магнитном поле, в котором сама магнитная $f$-система еще далека от насыщения. При малой мощности накачки концентрация фотоэлектронов мала, поэтому среднее расстояние между поляронами существенно больше их радиуса, следовательно, поляроны не взаимодействуют между собой и гистерезис в фотоиндуцированной намагниченности не возникает.

Первые исследования фотоиндуцированного эффекта Фарадея, обусловленного образованием магнитных поляронов, были проведены в антиферромагнетике EuTe $[19,20]$ и метамагнетике EuSe [21]. Результаты показали, что фотоиндуцированные магнитные поляроны представляют собой суперпарамагнитный ансамбль квазичастиц с большим магнитным моментом $10^{2}-10^{3} \mu_{\mathrm{B}}$, где $\mu_{\mathrm{B}}-$ магнетон Бора. Для полного намагничивания такого ансамбля достаточно небольшого магнитного поля $\sim 50 \mathrm{mT}$. Радиус магнитного полярона $\sim 3 \mathrm{~nm}$ велик по сравнению с постоянной решетки $\sim 0.6 \mathrm{~nm}$. За счет сильного $d-f$-обменного взаимодействия фотовозбужденный электрон поляризует более тысячи атомов европия, спины которых выстраиваются вдоль спина фотоэлектрона.

Поведение суперпарамагнитного ансамбля квазичастиц хорошо описываться функцией Ланжевена для парамагнитной системы в классическом пределе. Таким образом, фотоиндуцированную эллиптичность $E_{K}$, связанную с ансамблем магнитных поляронов, можно описать уравнением

$$
E_{K}=E_{K}^{S a t} L(x)
$$

где $E_{K}^{S a t}-$ керровская эллиптичность в поле насыщения, $L(x)$ - функция Ланжевена

$$
L(x)=\operatorname{coth}(x)-\frac{1}{x} .
$$

Здесь $x=\frac{\mu_{P o l} B}{k_{\mathrm{B}} T}, \mu_{P o l}-$ магнитный момент полярона, $B$ - магнитное поле, $k_{\mathrm{B}}$ - постоянная Больцмана, $T-$ температура.

В результате аппроксимации экспериментальных данных уравнениями (1) и (2) был определен магнитный момент полярона $\mu_{P o l} \sim 3000 \mu_{\mathrm{B}}$. До настоящего времени наибольший магнитный момент полярона был зафиксирован в селениде европия EuSe [21], он составил около $6000 \mu_{\mathrm{B}}$. Однако этот результат был получен для температуры $4.7 \mathrm{~K}$, а при температуре $14 \mathrm{~K}$ магнитный момент поляронов в EuSe составил менее $600 \mu_{\mathrm{B}}$, что в 5 раз меньше полученного нами магнитного момента полярона в сульфиде европия при данной температуре.

На рис. 2, $b$ дана зависимость фотоиндуцированной керровской эллиптичности $E_{K}^{S a t}$ в поле насыщения от частоты модуляции. Большое время жизни фотовозбужденных поляронов приводит к тому, что фотоиндуцированный эффект уменьшается при увеличении частоты модуляции накачки. Следуя процедуре, описанной в [20], можно определить время жизни полярона, которое составило $\tau_{P o l}=13 \mu \mathrm{s}$. Эта величина сравнима с временем жизни полярона в антиферромагнитном EuTe, в котором $\tau_{\text {Pol }}=15 \mu \mathrm{s}[20]$.

В отличие от антиферромагнитных EuTe и EuSe, в которых гигантские фотоиндуцированные поляроны наблюдались в широком диапазоне температур вплоть до $130 \mathrm{~K}[19,21]$, в ферромагнитном EuS фотоиндуцированный сигнал наблюдался лишь в интервале температур от 12 до $18 \mathrm{~K}$ вблизи температуры Кюри $T_{\mathrm{C}}=16 \mathrm{~K}$, см. рис. 3,a. Поскольку в ферромагнитном $\mathrm{EuS}$ условия для существования поляронов еще менее благоприятны, чем в антиферромагнетиках EuTe и EuSe, фотоиндуцированный магнетизм в $\mathrm{EuS}$ может быть обусловлен фотоэлектронами, захваченными на локальные уровни дефектов. Локализация электрона приводит к возникновению дополнительного $d-f$-обменного взаимодействия [22], а также может влиять на магнитную анизотропию, что проявляется через изменение магнитной восприимчивости кристалла. При температуре вблизи $T_{\mathrm{C}}$ 


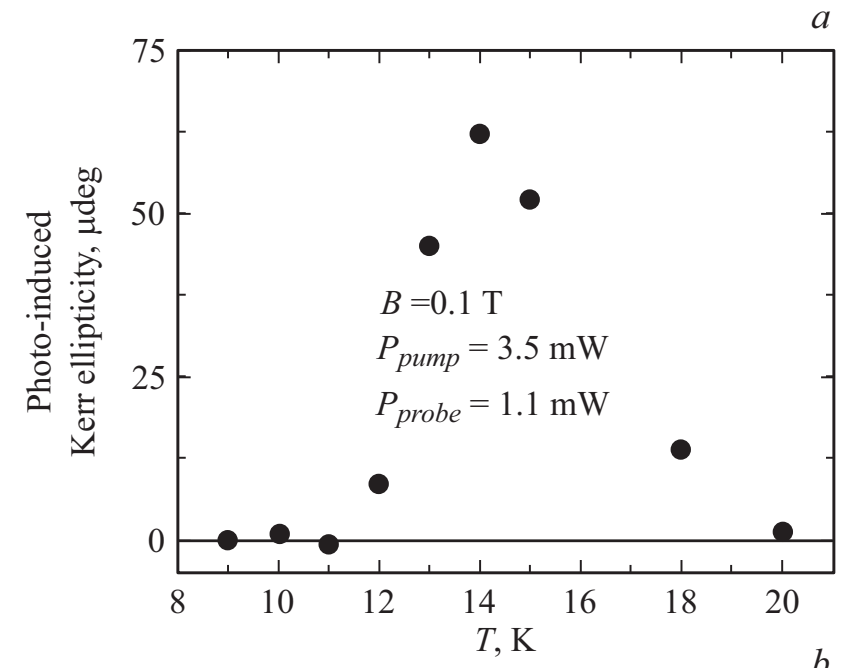

время спиновой релаксации $\sim 19 \mathrm{ps}$ в магнитном поле для возбужденных долгоживущих состояний $4 f^{6} X^{1}$, которые отвечают за образование фотоиндуцированных магнитных поляронов в ЕuТе. В [21] было напрямую измерено время нарастания фотоиндуцированной намагниченности в $\mathrm{EuSe}$, которое составило $62 \mathrm{ps}$. В настоящей работе время образования фотоиндуцированных магнитных поляронов в EuS не измерялось, но в силу схожести зонных структур халькогенидов европия $\mathrm{EuX}$ можно предположить, что оно составляет десятки пикосекунд. Этот вывод также согласуется по порядку величины с временем $200 \mathrm{ps}$ формирования магнитных поляронов в разбавленных магнитных полупроводниках [25].

С увеличением мощности накачки наблюдался переход от линейного роста величины фотоиндуцированного сигнала $E_{K}^{S a t}$ к более медленному, с его последующим насыщением (рис. $3, b$ ). Поскольку глубина области формирования магнитооптического эффекта Керра для зондирующего луча не зависит от мощности луча накачки, прекращение роста фотоиндуцированного сигнала свидетельствует о насыщении концентрации поляронов. Величина максимальной концентрации поляронов может быть получена из величины насыщенной фотоиндуцированной керровской эллиптичности (см. рис. $3, b$ ). Сравнивая насыщенную фотоиндуцированную эллиптичность с эллиптичностью полярного эффекта Керра в EuS при той же температуре, которая составила $2.4 \mathrm{deg}$, и нормируя на концентрацию атомов европия $n_{\mathrm{Eu}}=4 / a^{3}$, где $a=0.6 \mathrm{~nm}$ - параметр решетки, и магнитный момент атома европия $\mu_{\mathrm{Eu}}=7.9 \mu_{\mathrm{B}}$, можно получить для поляронов с магнитным моментом $3000 \mu_{\mathrm{B}}$ максимальную концентрацию $n_{D}=2 \cdot 10^{15} \mathrm{~cm}^{-3}$. В [19] также наблюдалось насыщение концентрации поляронов в ЕuТе на уровне $4.5 \cdot 10^{15} \mathrm{~cm}^{-3}$, и это было связано с фотонасыщением дефектов в области проникновения света накачки. Таким образом, насыщение фотоиндуцированного сигнала с ростом накачки согласуется с предположением о локализации фотоэлектронов на дефектах в этой области. Хотя EuS обладает ферромагнитным типом упорядочения, и условия для существования магнитных поляронов менее благоприятны, чем в $\mathrm{EuSe}$ и $\mathrm{EuTe}$, в настоящей работе удалось надежно установить основные параметры магнитных поляронов - магнитный момент, максимальную концентрацию, среднее время жизни и температурную область существования.

\section{4. Заключение}

концов тоже пропадает. Верхняя температурная граница области существования поляронов совпадает с температурой начала сильного сдвига края поглощения $\mathrm{EuS}$ в красную область спектра [17]. В [23] была разработана квантово-механическая модель для эффекта Фарадея в $\mathrm{EuTe}$ и было рассчитано фотоиндуцированное фарадеевское вращение, обусловленное магнитными поляронами. Разработанная квантово-механическая модель для эффекта Фарадея также может быть использована для описания фотоиндуцированного эффекта Керра, обнаруженного в EuS в данной работе. В [24] было определено

Исследование фотоиндуцированного эффекта Керра в объемном образце сульфида европия $\mathrm{EuS}$ показало, что данный материал может быть намагничен посредством оптического возбуждения магнитных поляронов с предельной концентрацией $\sim 2 \cdot 10^{15} \mathrm{~cm}^{-3}$. Намагниченность возникает в результате локализации фотоэлектронов в зоне проводимости, которые вследствие сильного $d-f$ обменного взаимодействия поляризуют соседние спины решетки и формируют магнитные поляроны. 
Магнитный момент полярона при температуре $14 \mathrm{~K}$ составил $\sim 3000 \mu_{\mathrm{B}}$, что в 5 раз больше магнитного момента полярона в EuSe при данной температуре [21]. Поляроны образуют суперпарамагнитный ансамбль со средним временем жизни около $13 \mu \mathrm{s}$. Они могут возбуждаться в узком диапазоне температур $12-18 \mathrm{~K}$, в котором происходит наиболее сильный красный сдвиг края фундаментального поглощения для EuS.

Сравнение результатов данной работы с исследованиями поляронов в антиферромагнитной группе халькогенидов европия EuTe [19] и EuSe [21] позволяет заключить, что в ферромагнитном $\mathrm{EuS}$ условия для существования магнитных поляронов являются другими. Время жизни и предельная концентрация поляронов в EuS сравнимы с этими величинами в EuTe, что указывает на локализацию фотоэлектронов на примесях или дефектах материала. Магнитный момент поляронов в $\mathrm{EuS}$ существенно больше чем магнитный момент поляронов в EuTe и EuSe при заданной температуре. Таким образом, полученные экспериментальные данные однозначно доказывают возможность фотовозбуждения магнитных поляронов в ферромагнитном полупроводнике EuS.

\section{Благорадности}

Авторы благодарят Р.В. Писарева за полезные консультации и В.В. Каминского за предоставленный образец EuS.

\section{Финансирование работы}

Авторы благодарят за финансовую поддержку по проектам РФФИ (19-52-12063 и 19-52-12066) и программы Президиума РАН № 5.

\section{Конфликт интересов}

Авторы заявляют, что у них нет конфликта интересов.

\section{Список литературы}

[1] M. Atatüre, D. Englund, N. Vamivakas, S.-Y. Lee, J. Wrachtrup. Nature Rev. Mater. 3, 38 (2018).

[2] M. Matsubara, A. Schroer, A. Schmehl, A. Melville, C. Becher, M. Trujillo-Martinez, D.G. Schlom, J. Mannhart, J. Kroha, M. Fiebig. Nature Commun. 6, 6724 (2015).

[3] A. Samanta, I.L. Medintz. Nanoscale 8, 9037 (2016).

[4] P. Wachter in Handbook on the Physics and Chemistry of Rare Earths. / K.A. Gschneider, L.R. Eyring. North-Holland, Amsterdam (1979).

[5] B. Kaminski, M. Lafrentz, R.V. Pisarev, D.R. Yakovlev, V.V. Pavlov, V.A. Lukoshkin, A.B. Henriques, G. Springholz, G. Bauer, E. Abramof, P.H.O. Rappl, M. Bayer. Phys. Rev. Lett. 103, 057203 (2009).

[6] M. Matsubara. Appl. Sci. 9, 948 (2019).

[7] D. Floess, J.Y. Chin, A. Kawatani, D. Dregely, H.-U. Habermeier, T. Weiss, H. Giessen. Light Sci. Appl. 4, e284 (2015).
[8] V.N. Kats, S.G. Nefedov, L.A. Shelukhin, P.A. Usachev, D.V. Averyanov, I.A. Karateev, O.E. Parfenov, A.N. Taldenkov, A.M. Tokmachev, V.G. Storchak, V.V. Pavlov. Appl. Mater. Today. 19, 100640 (2020).

[9] A. Schmehl, V. Vaithyanathan, A. Herrnberger, S. Thiel, C. Richter, M. Liberati, T. Heeg, M. Röckerath, L.F. Kourkoutis, S. Mühlbauer, P. Böni, D.A. Muller, Y. Barash, J. Schubert, Y. Idzerda, J. Mannhart, D.G. Schlom. Nature Mater. 6, 882 (2007).

[10] D.V. Averyanov, Y.G. Sadofyev, A.M. Tokmachev, A.E. Primenko, I.A. Likhachev, V.G. Storchak. ACS Appl. Mater. Interf. 7, 6146 (2015).

[11] A.G. Swartz, P.M. Odenthal, Y. Hao, R.S. Ruo, R.K. Kawakami. ACS Nano 6, 10063 (2012).

[12] H. Zhang, Y. Ma, H. Zhang, X. Chen, S. Wang, G. Li, Y. Yun, X. Yan, Y. Chen, F. Hu, J. Cai, B. Shen, W. Han, J. Sun. Nano Lett. 19, 1605 (2019).

[13] Э.Л. Нагаев. Физика магнитных полупроводников. Наука, M. (1979). 432 c.

[14] G. Güntherodt. Phys Condens Matter 18, 37 (1974).

[15] Э.Л. Нагаев. Письма в ЖЭТФ 6, 484 (1967).

[16] M. Umehara. Phys. Rev. B 52, 8140 (1995).

[17] P. Wachter. CRC Crit. Rev. Sol. State Sci. 3, 189 (1972).

[18] A. Mauger, D.L. Mills. Phys. Rev. B 31, 8024 (1985).

[19] A.B. Henriques, A.R. Naupa, P.A. Usachev, V.V. Pavlov, P.H.O. Rappl, E. Abramof. Phys. Rev. B 95, 045205 (2017).

[20] A.B. Henriques, G.D. Galgano, P.H.O. Rappl, E. Abramof. Phys. Rev. B 93, 201201(R) (2016).

[21] A.B. Henriques, X. Gratens, P.A. Usachev, V.A. Chitta, G. Springholz. Phys. Rev. Lett. 120, 217203 (2018).

[22] В.Ф. Коваленко, Э.Л. Нагаев. УФН 148, 561 (1986).

[23] A.B. Henriques, P.A. Usachev. Phys. Rev. B 96, 195210 (2017).

[24] V.V. Pavlov, R.V. Pisarev, S.G. Nefedov, I.A. Akimov, D.R. Yakovlev, M. Bayer, A.B. Henriques, P.H.O. Rapp, E. Abramof. J. Appl. Phys. 123, 193102 (2018).

[25] D.D. Awschalom, J.-M. Halbout, S. von Molnar, T. Siegrist, F. Holtzberg. Phys. Rev. Lett. 55, 1128 (1985).

Редактор К.В. Емцев 